\title{
Autoevaluación de habilidades clínicas básicas en médicos recién egresados de la Facultad de Medicina, Universidad de Chile
}

\author{
Teresa Millán Ka, Mariely Ercolano F, Marcela Pérez A, \\ Cristián Fuentes $F$.
}

Self perception of clinical competences declared by recently graduated physicians of the University of Chile

\begin{abstract}
Background: The new curriculum of the University of Chile School of Medicine includes the evaluation not only of knowledge and skills, but also abilities and attitudes. Aim: To measure the self perceived level of basic clinical competences (BCC) declared by recently graduated physicians. Material and methods: A self evaluation survey was designed, based on the proposed objectives of the Faculty Curriculum Committee and on an instrument used in Spanish Universities. It contained 194 questions and the possible answers were: 1 . I know what it is and it has been explained to me; 2 . I have seen it done; 3 . I have done it before under supervision; 4 . I would be capable to do it under any circumstance. It was applied confidentially to 50 of a total of 170 recently graduated physicians. Results: Perception of BCC for the diagnosis of most common diseases was felt as satisfactory. History taking and physical examination were also considered as achieved skills. Deficiences were found in practical aspects of nursing care, obstetric and gynecological abilities and reanimation procedures. Discussion: Answers may be biased considering that the survey was a self assessment procedure. However, results provide sound orientation to detect strengths and weaknesses of delivered education. Achievement of BCC is proportional to clinical practice opportunities as a student (Rev Méd Chile 2007; 135: 1479-86).
\end{abstract}

(Key words: Clinical competence; Curriculum; Education, medical)

Recibido el 23 de octubre, 2006. Aceptado el 29 de agosto, 2007.

Departamento de Pediatría y Cirugía Infantil Occidente, Facultad de Medicina, Universidad de Chile. Santiago de Chile.

a Matrona.

Correspondencia a: Teresa Millán. Avenida Las Palmeras 299 Interior Quinta Normal, Santiago. Casilla 33052. Correo 33. Tel: 6816630 - 6816298. E mail: tmillan@med.uchile.cl 
$\mathrm{L}$ a misión reconocida por la Facultad de Medicina de la Universidad de Chile, incluye "formar profesionales de la salud, de gran capacidad, con sólida formación ética, espíritu de servicio y compromiso con el bienestar de la comunidad para contribuir a elevar la calidad de vida de la población...".

De acuerdo con ella, la Facultad, en un proyecto de planificación estratégica dirigido a fortalecer y promover la calidad y excelencia académicas, inició en 1998 un cambio curricular innovando planes y programas de la Carrera de Medicina ${ }^{1,2}$. Dichos cambios son acordes con las nuevas tendencias en educación médica que plantean las necesidades educativas en forma mucho más compleja, debido a los avances científicos, el dominio de las tecnologías de punta y el acceso y uso de la informática. Todo esto constituye un reto para el sistema educativo en su tarea de preparar las generaciones profesionales del nuevo milenio.

Coexisten otros desafíos, igualmente importantes, como las demandas formativas para el desarrollo social de la persona. Esto significa educar para la aceptación de la diversidad, creatividad, cooperación y la capacidad de generar un progreso personal y social basado en una ética humanista.

En este contexto, el sujeto que aprende debe ser concebido como una totalidad (conocimiento, afecto y voluntad) y como constructor de sus propios conocimientos ${ }^{2-6}$.

En este marco, el currículo innovado contempla la adquisición de competencias clínicas, definidas como "un conjunto complejo de habilidades del estudiante, que dicen relación no sólo con sus conocimientos, habilidades y destrezas específicas, sino que con hábitos, actitudes, conductas y valores médicos ${ }^{4,7}$.

Las competencias clínicas definidas en el currículo son nueve y se espera que el estudiante de medicina las adquiera y desarrolle durante la carrera en tres niveles de ejecución: inicial ( $1^{\circ}$ y $2^{\circ}$ años), intermedio ( $3^{\circ}$ a $5^{\circ}$ años) y avanzado ( $6^{\circ}$ y 7o años).

Entre las competencias clínicas más importantes figuran: comunicación efectiva; conciencia de fortalezas y debilidades personales; razonamiento ético; solución de problemas; aprendizaje permanente; habilidades clínicas básicas; diagnóstico, manejo y prevención; aplicación de las ciencias básicas y clínicas para guiar el tratamiento del paciente y el contexto social y comunitario de la atención de salud ${ }^{7-10}$.

En este contexto de innovación educacional, se planteó el objetivo de conocer el nivel de auto percepción de competencias logrado en el conjunto de las habilidades clínicas básicas (HCB) manifestadas por egresados sometidos al nuevo currículo mediante una auto evaluación. Además, se contrastó esta percepción con lo planteado por la reforma curricular ${ }^{1}$. Esta información permitirá hacer propuestas para retroalimentar el proceso de enseñanza aprendizaje y fortalecer áreas discordantes o deficitarias.

\section{Personas y método}

Estudio descriptivo en el cual se diseñó, validó y aplicó una encuesta -confidencial, voluntaria, con consentimiento informado y anónima- de autoevaluación de HCB al total de médicos recién egresados el año 2005 de dos de los cinco Campus de la Universidad de Chile: Oriente (n =25) y Occidente $(n=40)$. Se intentó estudiar a 65 egresados (38,3\% del total de 170 que egresaron de los cinco Campus y se obtuvo respuesta de 50 de ellos; lo que corresponde al 29,4\%).

Para el diseño de la encuesta se utilizó como base el documento "Evaluación de las Competencias de los Estudiantes de la Carrera de Medicina", del Comité de Currículum de la Facultad de Medicina (UPEM) ${ }^{7}$ y se adaptó otro instrumento validado en dos Universidades españolas ${ }^{8-9}$, el cual contiene detalladamente las habilidades requeridas para el desempeño de las habilidades clínicas básicas exigidas por el currículo de las universidades respectivas.

El formato aplicado llegó a 194 preguntas, divididas en dos partes. La primera aborda las HCB con 85 preguntas y la segunda, los procedimientos clínicos y exámenes de laboratorio con 108 preguntas. La secuencia de temas fue: habilidades de anamnesis y examen físico, realización e interpretación de exámenes de laboratorio básico e imágenes, procedimientos clínicos básicos y especializados, búsqueda de información bibliográfica, habilidades comunicacionales (presentación de casos clínicos, comunicación de la información en la relación médico paciente), 
incluyéndose en esta última la realización de informes de lesiones al Juzgado.

Para cada habilidad o procedimiento el cuestionario propuso las siguientes categorías de respuestas: 1) Sé lo que es y me lo han explicado; 2) Lo he visto hacer; 3) Lo he practicado bajo supervisión; 4) Sería capaz de realizarla siempre y en cualquier circunstancia.

El nivel de logro se evaluó considerando los parámetros de evaluación y niveles de ejecución planteados para estudiantes de la Carrera de Medicina $^{7}$. En todos ellos el nivel de competencia exigido por la Escuela de Medicina corresponde a la autonomía máxima, es decir, "ser capaz de efectuar siempre y en cualquier circunstancia la acción requerida". La única excepción en el nivel de competencia logrado lo constituyó la realización de procedimientos clínicos especializados en que se exige "haber observado la realización y eventualmente realizado bajo supervisión directa, conocer sus indicaciones, limitaciones y complicaciones"7.

El análisis de la información se realizó con el programa Epi Info 2002 expresándose los resultados en frecuencias relativas.

\section{Resultados}

No hubo rechazos entre quienes se contactaron pero $23 \%$ de quienes se incluyó en el diseño se encontraban en regiones extremas del país o el extranjero. Las respuestas tabuladas alcanzan al $29,4 \%$ del total de egresados de los cinco campus y al $76,9 \%$ de quienes se incluyó en el diseño.

Los resultados se presentan de acuerdo a la distribución porcentual alcanzada por los egresados para cada HCB autoevaluada, en orden decreciente, según el siguiente esquema:

Anamnesis y examen físico; realización e interpretación de exámenes de laboratorio básico; interpretación de exámenes especializados (laboratorio e imágenes); procedimientos clínicos básicos y especializados; habilidades comunicacionales y búsqueda bibliográfica.

En la anamnesis la totalidad refirió ser capaz de "obtener una historia clínica acabada en una variada gama de encuentros con pacientes siempre y en cualquier circunstancia".
En el examen físico las áreas con mayores porcentajes de habilidades satisfactorias fueron: piel y sistema linfático, cardiovascular, genital, abdominal, pulmonar, neurológico, de la región del cuello, aparato locomotor, oftalmológico y otorrinolaringológico. El aspecto con las menores cifras fue el examen mental (Tabla 1).

En los exámenes de laboratorio básico, en que el estudiante debe ser capaz de "realizar personalmente, saber interpretarlos y conocer indicaciones y contraindicaciones ${ }^{\prime 7}$ hubo logro óptimo sólo en el hemoglucotest. Los niveles más bajos se encontraron en el ámbito microbiológico: siembra de muestra bacteriológica, tinción de Gram, frotis de bacterias alcohol ácido resistente y prueba de látex en líquido céfalo raquídeo (Tabla 2).

Los egresados deberían ser capaces de "interpretar los informes escritos de procedimientos clínicos y exámenes de laboratorio, conocer sus indicaciones, limitaciones y complicaciones" ${ }^{7}$. En estas competencias ningún ítem fue totalmente logrado. El mejor resultado lo alcanzó la gasometría arterial y los peores fueron los estudios electrofisiológicos (electroencefalograma y electromiograma) (Tabla 3).

\section{Tabla 1. Anamnesis y examen físico, autoevaluación de logro estimado*}

\begin{tabular}{|lr|}
\hline & $(\%)$ \\
\hline Anamnesis & 100,0 \\
Piel y linfático & 94,0 \\
Cardiovascular & 93,1 \\
Genital & 91,0 \\
Abdomen & 90,3 \\
Pulmonar & 90,2 \\
Neurológico & 89,4 \\
Cuello & 86,0 \\
Locomotor & 83,5 \\
Oftalmológico & 80,5 \\
Otorrinolaringológico & 75,0 \\
Estado mental & 60,7 \\
\hline
\end{tabular}

*"El estudiante competente es capaz de obtener una historia clínica acabada en una variada gama de encuentros con pacientes" UPEM . 
Tabla 2. Laboratorio básico, autoevaluación de logro estimado*

\begin{tabular}{|lr|}
\hline & $\%$ \\
\hline Hemoglucotest & 100 \\
Hematocrito y hemoglobina & 93,9 \\
Radiografía de abdomen simple & 93,9 \\
Radiografía de tórax simple & 91,8 \\
Examen de orina & 89,8 \\
ECG & 89,6 \\
Radiografía simple de esqueleto & 87,8 \\
Papanicolau & 83,7 \\
Bilirrubina & 81,6 \\
Frotis sanguíneo & 77,6 \\
Sangre oculta en deposiciones & 77,1 \\
Siembra de muestra bacteriológica & 69,4 \\
Tinción de Gram & 63,3 \\
Frotis bacterias alcohol resistentes & 36,7 \\
Pruebas en látex de LCR & 28,6 \\
\hline
\end{tabular}

*"El estudiante debe ser capaz de realizar personalmente, saber interpretarlos y conocer indicaciones y contraindicaciones" UPEM.

En la interpretación de informes de imágenes se encontró que sólo dos de ellos, mamografía básica y ecotomografía mamaria, y estudios radio-
Tabla 3. Interpretación de informes escritos, autoevaluación de logro estimado*

\begin{tabular}{|lc|}
\hline & $(\%)$ \\
\hline Gasometría arterial & 91,8 \\
Química sanguínea & 83,0 \\
Estudios de coagulación & 81,6 \\
Examen parasitológico de deposiciones & 77,6 \\
Estudios de líquido céfalo raquídeo & 77,1 \\
Cultivo y sensibilidad microbiana & 75,5 \\
Hematología & 73,5 \\
Citología & 69,4 \\
Informes de anatomía patológica & 65,3 \\
Estudios vasculares no invasivos & 63,3 \\
Niveles de droga y antibióticos & 61,2 \\
Test de función respiratoria & 59,2 \\
Monitoreo Holter & 56,1 \\
Estudios endocrinos & 49,0 \\
Inmunológicos & 30,6 \\
Genéticos & 28,6 \\
Electroencefalograma & 26,5 \\
Electromiograma & 26,5 \\
\hline
\end{tabular}

*"El estudiante debe ser capaz de interpretar los informes escritos de los siguientes procedimientos clínicos y exámenes de laboratorio, conocer sus indicaciones, limitaciones y complicaciones" UPEM.

lógicos de contraste del tubo digestivo con bario, fueron logrados por más de la mitad de los egresados (Tabla 4).

Tabla 4. Interpretación de informes escritos imagenológicos, autoevaluación de logro estimado*

\begin{tabular}{|lc|}
\hline & $(\%)$ \\
\hline Mamografía básica y ecotomografía de mama & 63,3 \\
Estudios radiológicos de contraste del tubo digestivo con bario & 53,1 \\
Estudios de contraste renal intravenoso & 49,0 \\
Ultrasonido de pelvis, abdominal y de masas quísticas & 49,0 \\
Cintigrafías & 46,9 \\
Tomografía axial computada & 42,9 \\
Resonancia nuclear magnética & 40,8 \\
Angiografía básica & 40,8 \\
Ultrasonido cardíaco y Doppler & 30,6 \\
\hline
\end{tabular}

*"El estudiante debe saber interpretar los informes escritos de los siguientes procedimientos clínicos y exámenes de laboratorio, sus indicaciones, limitaciones y complicaciones" UPEM. 


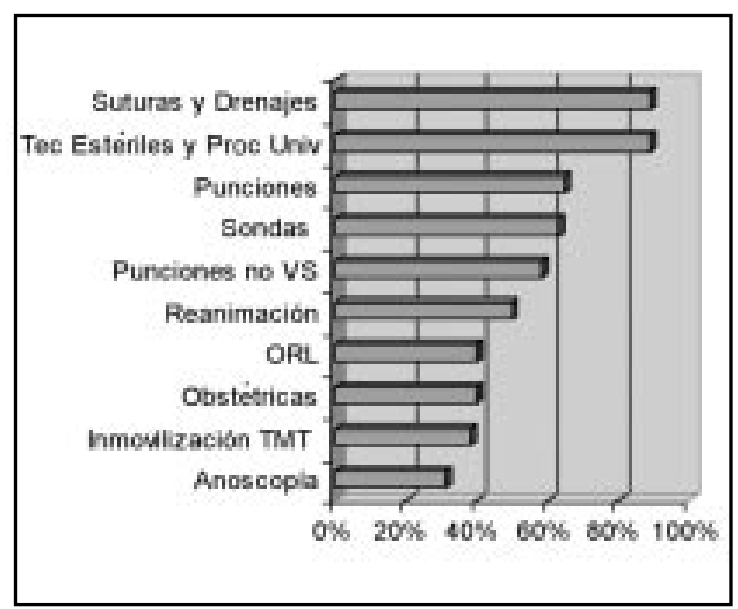

Figura 1. Procedimientos clínicos básicos, agrupados según área de destreza. Autoevaluación de logro estimado en porcentaje promedio.
En procedimientos clínicos básicos, se exige a los alumnos "máxima autonomía". Sin embargo, los resultados obtenidos son menores a los esperados. Dada su importancia se presentan en detalle y agrupando las punciones no venosas, procedimientos de reanimación y procedimientos obstétricos (Figura 1 y Tabla 5).

En relación a procedimientos clínicos especializados, se espera que el estudiante concluya su formación habiendo, por lo menos, "observado la realización y eventualmente realizado bajo supervisión directa, y conozca sus indicaciones, limitaciones y complicaciones"7. Este objetivo se logró en reducción de fracturas por la totalidad de los egresados, mientras que procedimientos como pericardiocentesis y marcapasos transitorio transvenoso alcanzaron menos de la mitad del logro esperado (Tabla 6).

Tabla 5. Procedimientos clínicos básicos, autoevaluación de logro estimado*

\begin{tabular}{|lc|}
\hline & $(\%)$ \\
\hline Sutura heridas & 93,9 \\
Manejo de técnicas estériles & 89,8 \\
Aplicar precauciones universales & 87,8 \\
Incisión y drenaje de lesiones superficiales & 83,7 \\
Colocar inyecciones intradérmica, subcutánea, intramuscular, intravenosa & 81,6 \\
Colocación de sonda Foley & 75,0 \\
Paracentesis abdominal & 73,5 \\
Iniciar una infusión endovenosa & 67,3 \\
Manejo ventilatorio básico & 65,3 \\
Resucitación cardiorespiratoria básica & 63,3 \\
Realizar control de hemorragia externa masiva & 63,3 \\
Realizar una punción venosa incluyendo toma de hemocultivos & 59,2 \\
Realizar inmovilización cervical de emergencia & 57,0 \\
Aplicación de férulas de emergencia & 55,0 \\
Intubación nasogástrica & 51,0 \\
Punción lumbar & 51,0 \\
Toracocentesis & 51,0 \\
Atención inmediata del recién nacido y apego & 49,0 \\
Colocación de yeso en fracturas cerradas sin desplazamiento & 46,9 \\
Realizar frotis faríngeo & 46,8 \\
Colocación de tubo endotraqueal & 44,9 \\
Laringoscopia indirecta & 33,3 \\
Anoscopia & 31,3 \\
Atención parto vaginal normal & 30,6 \\
Realizar denudación venosa & 12,5 \\
Realizar punción cricotiroidea & 10,4 \\
\hline
\end{tabular}

*"El estudiante debe tener competencia para realizar personalmente procedimientos clínicos de rutina y exámenes de laboratorio básico, saber interpretarlos y conocer sus indicaciones, complicaciones y limitaciones" UPEM. 
Tabla 6. Procedimientos clínicos especializados, autoevaluación de logro estimado*

\begin{tabular}{|lc|}
\hline & $(\%)$ \\
\hline Reducción de fracturas & 100,0 \\
Tonometría ocular & 95,9 \\
Punción y aspiración articular & 93,9 \\
Tinción ocular con fluoresceína & 89,9 \\
Punción arterial & 87,8 \\
Atención de parto distócico & 87,8 \\
Cardioversión eléctrica & 87,7 \\
Punción de masa mamaria y tiroídea & 87,5 \\
Laringoscopia directa & 85,8 \\
Endoscopia digestiva alta & 85,7 \\
Aspiración transtraqueal & 85,4 \\
Manejo de alimentación parenteral & 79,6 \\
Biopsia cutánea & 77,6 \\
Endoscopia digestiva baja & 77,5 \\
Colocación de catéter venoso central & 77,5 \\
Broncoscopia flexible & 77,5 \\
Remoción de lesiones cutáneas & 75,5 \\
Test de esfuerzo & 73,5 \\
Colocación de cánula arterial & 71,4 \\
Toma de biopsias endoscópicas & 67,3 \\
Diálisis peritoneal & 65,3 \\
Aspiración de médula ósea & 56,3 \\
Lavado peritoneal diagnóstico & 53,1 \\
Colocación de catéter de Swan-Ganz & 53,1 \\
Realización de biopsia renal y hepática percutánea & 51,0 \\
Pericardiocentesis & 47,0 \\
Marcapasos transitorio transvenoso & 42,8 \\
\hline
\end{tabular}

*"El estudiante debe haber observado la realización y eventualmente realizado bajo supervisión directa, conocer sus indicaciones, limitaciones y complicaciones" UPEM

Tabla 7. H abilidades comunicacionales y de búsqueda bibliográfica, autoevaluación de logro estimado

\begin{tabular}{|lr|}
\hline & $\%$ \\
\hline Usar el correo electrónico & 100,0 \\
Búsquedas en Medline (PubMed) & 79,6 \\
Búsquedas en bases bibliográficas basadas en la evidencia & 69,4 \\
Presentar un caso clínico & 100,0 \\
Realizar un informe clínico & 100,0 \\
Solicitar un consentimiento informado & 93,5 \\
Informar al paciente de su curso clínico & 95,9 \\
Informar a la familia del curso clínico del paciente & 95,9 \\
Informar a la familia de una mala noticia & 83,7 \\
Hacer un informe de lesiones al Juzgado & 30,6 \\
\hline
\end{tabular}


Los egresados manifestaron poseer un alto nivel de competencias comunicacionales. El uso adecuado del correo electrónico, presentación de casos clínicos y confección de informes clínicos fueron plenamente logrados. Contrasta, por sus bajos resultados, la entrega de malas noticias y la confección de un parte de lesiones al Juzgado (Tabla 7).

\section{DisCUSIÓN}

Nos parece importante hacer algunas consideraciones que permitirán una mejor comprensión de la discusión.

El grupo estudiado estuvo conformado por médicos recién egresados de la Facultad de Medicina de la Universidad de Chile, que distribuye sus alumnos en cinco campus clínicos, de acuerdo a cupos disponibles, lo que hace que el tamaño de los grupos sea diferente. Como se señalara en sujetos y método, por razones de recursos y tiempo, solo se encuestaron los Campus Oriente y Occidente, dejando para una segunda etapa los restantes. Los egresados habían completado satisfactoriamente el proceso de formación y evaluación del pregrado, rindiendo además el Examen Médico Nacional, prueba obligatoria para quienes egresan de la Carrera de Medicina de las Universidades Chilenas. Lo anterior fundamenta el proceso de evaluación interna y externa a que fueron sometidos los estudiantes. Esta investigación correspondió a un corte transversal al término de los estudios de pregrado, sin olvidar la importancia del postgrado en la formación del médico y su necesidad de perfeccionamiento continuo. La evaluación de competencias es un nuevo paradigma orientado a evaluar el producto final logrado con los programas de formación universitarios en el campo de la salud. Conocer parte de sus resultados permite retroalimentar el proceso de enseñanza aprendizaje de las escuelas, sus logros y eventuales falencias estimulando así su progreso educativo ${ }^{1,2,12}$. La auto evaluación es un elemento que se ubica en la cúspide de la pirámide del modelo de adquisición y logro de competencias, donde es necesario tener presente la existencia de un juicio o autocrítica de mayor o menor exigencia según cada individuo y que introduce, en consecuencia, un sesgo en los resultados, que hay que tener presente.
La HCB que destacó por su mayor satisfacción de logro fue la anamnesis, lo que contrasta con los bajos porcentajes de logro publicados en estudios similares en España-8. Este alto grado de autonomía podría corresponder a que en la Facultad se conserva $-y$ se ha podido conservarel aprendizaje del ars médica junto al paciente, a diferencia de los países europeos y de América del Norte, donde el aprendizaje de estas habilidades es, cada vez con mayor frecuencia, con simuladores o actores. La permanencia de la enseñanza "alrededor del paciente" se puede atribuir en forma considerable al modelo docente asistencial, que se siente casi como parte de la cultura de la atención de salud en el sistema público chileno.

Los egresados, además, señalaron un elevado nivel de habilidad con respecto a examen físico. Una explicación de esto sería el haber mantenido el ejercicio continuo de esta habilidad durante la formación del pregrado. Una excepción la constituyó el examen mental, situación que la Facultad está corrigiendo con la incorporación de un internado de psiquiatría a partir del año 2006. Sin embargo, es necesario enfatizar la necesidad de un nuevo enfoque, que otorgue a la salud mental la importancia que merece en la medicina del siglo XXI.

El nuevo currículum permite, desde el ingreso a la Carrera, el contacto directo entre alumnos, pacientes y familia ${ }^{1}$. Lo anterior, además de explicar las ventajas en la práctica del acto médico en sí, puede contribuir a los buenos resultados observados en las habilidades comunicacionales y de relación médico-paciente. La Escuela se ha esforzado en promover las competencias comunicacionales, dándoles énfasis e integrando asignaturas de comunicación a la malla curricular. La dificultad en la entrega de malas noticias probablemente responda a que este acto involucra el área emocional de los participantes, lo que depende no sólo de la educación formal, sino también de condiciones personales que facilitan o dificultan este acto.

En la realización e interpretación de exámenes de laboratorio básico llamó la atención que exámenes de uso frecuente, como bilirrubinemia, electrocardiograma y examen de orina, no fueran dominados por todos. Si bien el médico no realiza en su quehacer diario los exámenes de laborato- 
rio, su labor a la cabeza del equipo de salud le hace responsable de conocer a cabalidad la realización, interpretación, indicaciones y contraindicaciones de los exámenes que solicita, por lo menos los más frecuentes. Una razón que podría explicar el bajo rendimiento en esta área puede ser el aún imperante paradigma biomédico curativo y positivista. Éste, frente a la complejidad del individuo y sus sistemas biológicos en interacción, abarca áreas limitadas del conocimiento, fraccionando al ser humano en órganos y enfermedades. Lo anterior lleva a una tendencia a la excesiva especialización y sub especialización, que fomentaría en los alumnos una visión sesgada, concentrando sus esfuerzos en áreas de su mayor interés.

Es posible que las cifras bajas observadas en los procedimientos clínicos básicos y especializados, reflejen las oportunidades de práctica clínica, recursos disponibles, autonomía en la atención del paciente, marco médico-legal actual y normas de los Servicios de Salud y centros formadores ${ }^{9,10}$.

\section{REFERENCIAS}

1. Herskovic P. La reforma curricular de la Escuela de Medicina de la Universidad de Chile. Rev Chil Pediatr 2005; 76: 9-11.

2. Escuela de Medicina, Facultad de Medicina. Universidad de Chile www.med.uchile.cl. Consultado el 4 de junio 2007.

3. Harden R, Crosby Jr, Davis Mh, Friedman M. AmeE guide № 14: Outcome - based education (Part 5). From competency to meta competency: a model for the specification of learning outcomes. Med Teach 1999; 21: 546-52.

4. Brofenbrenner U. The Ecology of Human Development. Cambridge, MA: Harvard University press, 1979.

5. González F. Problemas Epistemológicos de la Psicología. México: Colegio de Ciencias y Humanidades. Universidad Nacional Autónoma de México, 1998.

6. Rosselot E. Realidad y proyecciones. Educación en ciencias de la salud en Chile. Anales de la Universidad de Chile, Sexta Serie 2006; 16: 71-96.
Sin embargo, el ente formador tiene una gran responsabilidad frente al logro de las competencias que conforman el perfil profesional del egresado $^{7}$, y debe garantizar las oportunidades y acceso a las experiencias y actividades de enseñanza aprendizaje.

Una propuesta para resolver esta demanda sería integrar aún más a los cinco Campus Clínicos de la Facultad en una red de enseñanza multicéntrica, fortaleciendo las unidades y rotando alumnos según posibilidad y oportunidad. Naturalmente sin abandonar la enseñanza en centros de menor complejidad, que permite al estudiante relacionarse más directamente con la patología prevalente.

La auto evaluación de HCB al final de los estudios de una promoción de alumnos formados con el nuevo currículo, permitió aproximarnos a conocer su auto percepción del logro obtenido, reconociendo fortalezas - para estimular su permanencia- y detectando debilidades para replantear tareas y modificar aspectos susceptibles de variación.

7. Universidad de Chile. Comité de Currículum de la Facultad de Medicina. Evaluación de las Competencias de los Estudiantes de la Carrera de Medicina, 2000.

8. Palés J, Vallés A, Cardellach F, Gomar C, Estrach M, Cost J, et al. Habilidades y procedimientos clínicos básicos a adquirir por los estudiantes de la Facultad de Medicina de la Universidad de Barcelona. Educ Med 2001; 4: 72-81.

9. Torres-Cisneros J, Camacho A, López-Miranda J Sánchez-Guijo P, Pérez F. El proceso docente de adquisición de habilidades clínicas en la Universidad de Córdova: una autoevaluación de los alumnos. Educ Med 2003; 6: 117-22.

10. Pacheco D, Gatica H, Kalinski S. Autoevaluación de fortalezas, debilidades y confianza de los médicos de atención primaria en el abordaje de enfermedades reumatológicas. Rev Méd Chil 2006; 134: 813-20.

11. Schonhaut L. Los desafíos de la enseñanza de pediatría ambulatoria en el contexto del Chile actual. Rev Chil Pediatr 2006; 77: 405-41.

12. Rosselot E. Planificando la educación médica para las próximas décadas. Rev Méd Chile 2001; 129: $1473-8$. 